\title{
Radiographic and Computed Tomographic Anatomy of the Donkey Carpus
}

\author{
Mohamed A.M. Alsafy ${ }^{1}$ Mahmoud H. El-Kammar ${ }^{2}$ Samir R. Nouh ${ }^{2}$ Howaida M. Abou-Ahmed ${ }^{2}$ \\ William Perez ${ }^{3}$ Noelia Vazquez ${ }^{3}$ Dina Swidan ${ }^{2}$
}

${ }^{1}$ Department of Anatomy and Embryology, Faculty of Veterinary Medicine, Alexandria University, Alexandria, Egypt

2 Department of Surgery, Faculty of Veterinary Medicine, Alexandria University, Alexandria, Egypt

${ }^{3}$ Division of Anatomy, Facultad de Veterinaria, Universidad de la República, Montevideo, Uruguay

Address for correspondence Mohamed A.M. Alsafy, PhD, Department of Anatomy and Embryology, Faculty of Veterinary Medicine, Alexandria University, 22758, Alexandria, Egypt (e-mail: safy73@yahoo.com).

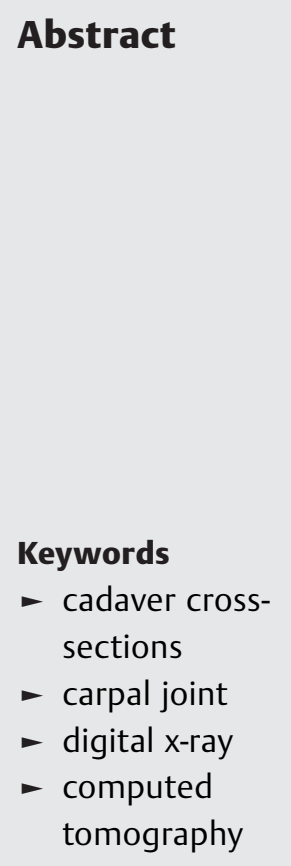

Introduction Joint diseases represent most of the musculoskeletal disorders. Therefore, the aim of the current study was to make a radiographic and computed tomographic analysis of the structures of the donkey carpus and investigate carpal joint affections. Materials and Methods The study was performed with the use of cross-sectional anatomy, digital X-ray and computed tomography (CT) scans. Twelve adult donkeys were used.

Results The results provide a full description of the bones and soft tissues of the carpus. The carpal joint was examined at many levels using different $\mathrm{CT}$ and X-ray planes. The carpus was studied through bone and soft-tissue windows that were compared with cadaver cross-sections for interpretation. The study revealed some joint affections that were detected by the CT scans but were unapparent in X-ray films, such as bony cysts, hemorrhagic bony cysts, old and microfractures, and bony sclerosis. Some normal anatomic variants were recorded during the examination of the CT scans to assist the equine practitioners that deal with the carpal joints of donkeys.

Conclusion Both imaging techniques are suitable for the examination of the carpus, and the selection of the technique is conditioned to many factors, like the type of tissue affected and economic reasons, such as the availability of the apparatus and the cost of the animal.

\section{Introduction}

The donkey is an important farming animal that is widely used by Egyptian farmers. They use donkeys as a cheap means of transportation and in farm work, so these animals play a significant role in the Egyptian economy. ${ }^{1}$ Equine practitioners should be aware of several significant features

(D) Mohamed A.M. Alsafy's ORCID is https://orcid.org/0000-0001-62364801.

received

September 5, 2018

accepted

June 10, 2019
DOI https://doi.org/

10.1055/s-0039-1693719. ISSN 2177-0298. that are specific to donkeys when they deal with them as a patients. $^{2}$

Joint diseases represent most of the musculoskeletal disorders. ${ }^{3-5}$ The joints normally function silently throughout life, but when joints fail, they cripple. ${ }^{6}$ Carpal injuries are a common cause of lameness in horses. Osteoarthritis is a group of disorders characterized by cartilage, bone and softtissue changes (Park et al, 1996). ${ }^{7}$ Furthermore, the ultimate goal in equines with joint disease is to make an accurate and early diagnosis to treat and prevent the ongoing deterioration of the disease. ${ }^{8}$

Copyright $\odot 2019$ by Thieme Revinter Publicações Ltda, Rio de Janeiro, Brazil 
Radiography has been commonly used to diagnose the bony and muscular affections of equine limbs due to many advantages, such as its low cost and easy accessibility ${ }^{9,10}$.The complexity of the carpus requires good-quality radiographs in order for the practitioners to make definitive radiographic interpretations. ${ }^{11}$ In general, the lesions are most easily visualized on one or two of the standard projections. ${ }^{11}$ Nevertheless, radiography is of limited value in the evaluation of soft tissue. Computed tomography (CT) and magnetic resonance imaging (MRI) are two modern techniques that enable a better evaluation of ligamentous, tendinous and synovial abnormalities. ${ }^{12,13}$ The disadvantages of these techniques are the high cost and the need for general anesthesia.

Computed tomography provides excellent transverse images and high spatial resolution without superimposition, which enables a better examination of the anatomy and abnormalities of the limb bones and soft tissues (Badawy, 2011). ${ }^{14}$ Moreover, CT is a useful tool to diagnose most injuries of the carpal bone, like bony sclerosis and fractures, as well as the carpal tendon sheath and its contents. ${ }^{15}$ In order to make the best interpretation of the CT images of equine limbs, the practitioners need adequate information of the transverse anatomy of the carpus to evaluate and investigate any pathological disorders. Computed tomography is also useful for the examination of complex bony structures, such as the skull, the spine and the joints. ${ }^{16}$

The purpose of the present study was to provide a detailed radiographic and tomographic anatomical reference for the donkey carpus in normal and pathological conditions.

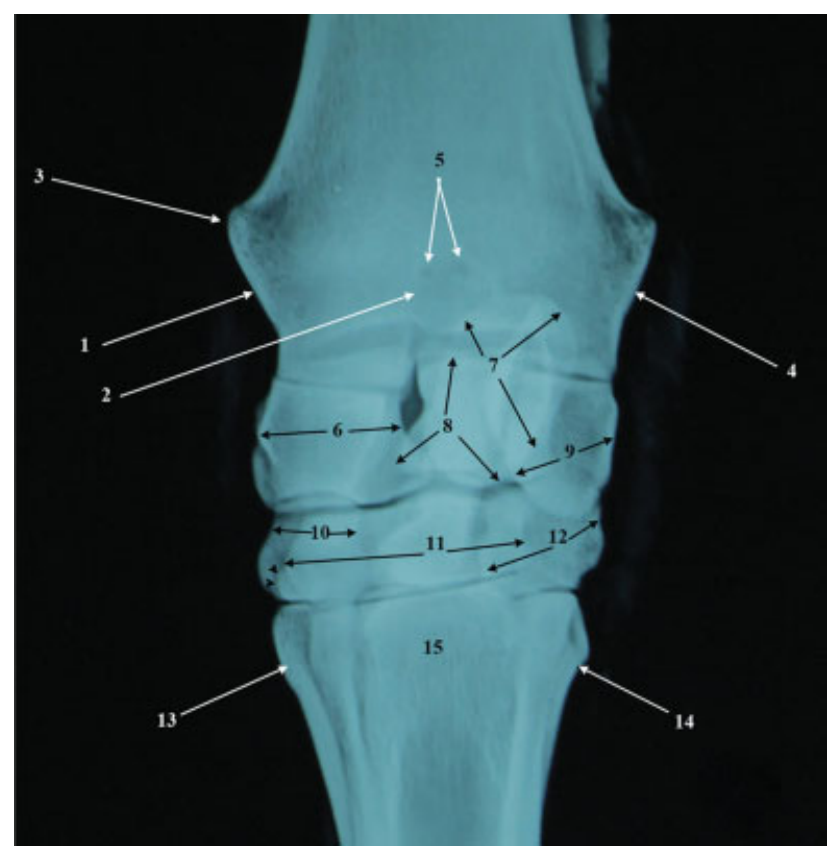

Fig. 1 Dorsopalmar radiograph of the left carpal joint. 1. Medial styloid process. 2. Lateral border of the medial styloid process. 3. Projection at the proximo-medial aspect of the medial styloid process. 4 . Lateral styloid process. 5. Vascular channels. 6. Radial carpal bone. 7. Accessory carpal bone. 8. Intermediate carpal bone. 9. Ulnar carpal bone. 10. Second carpal bone. 11. Third carpal bone. 12. Fourth carpal bone. 13. Second metacarpal bone. 14. Fourth metacarpal bone. 15. Third metacarpal bone. Note the border of the first carpal bone (black arrowheads).

\section{Materials and Methods}

\section{Animals}

The present study was performed on twelve mature donkeys of both sexes, which were deemed healthy after examination at the veterinary clinic of the Faculty of Veterinary Medicine at Alexandria University. All animals were deeply anesthetized with pentobarbital and thiopental sodium. One donkey died due to causes not related to our study, and was used for crosssection anatomical slices; its thoracic limb was disarticulated at the elbow joint, and the stumps were wrapped with a plastic sheath to prevent contamination of the working area.

The present study followed the guidelines for the care and use of animals, and was approved by the Animal Welfare and Ethics Committee of the Faculty of Veterinary Medicine at Alexandria University, according to Egyptian law.

\section{Digital Radiography}

All carpal joints were subjected to X-ray beams - using a Toshiba 500-ml X-ray machine (Toshiba, Minato, Tokyo, Japan) - with an output of $80 \mathrm{Kv}$ and $320 \mathrm{mAs}$ in the dorsopalmar, lateromedial and dorsolateral-palmaromedial oblique views.

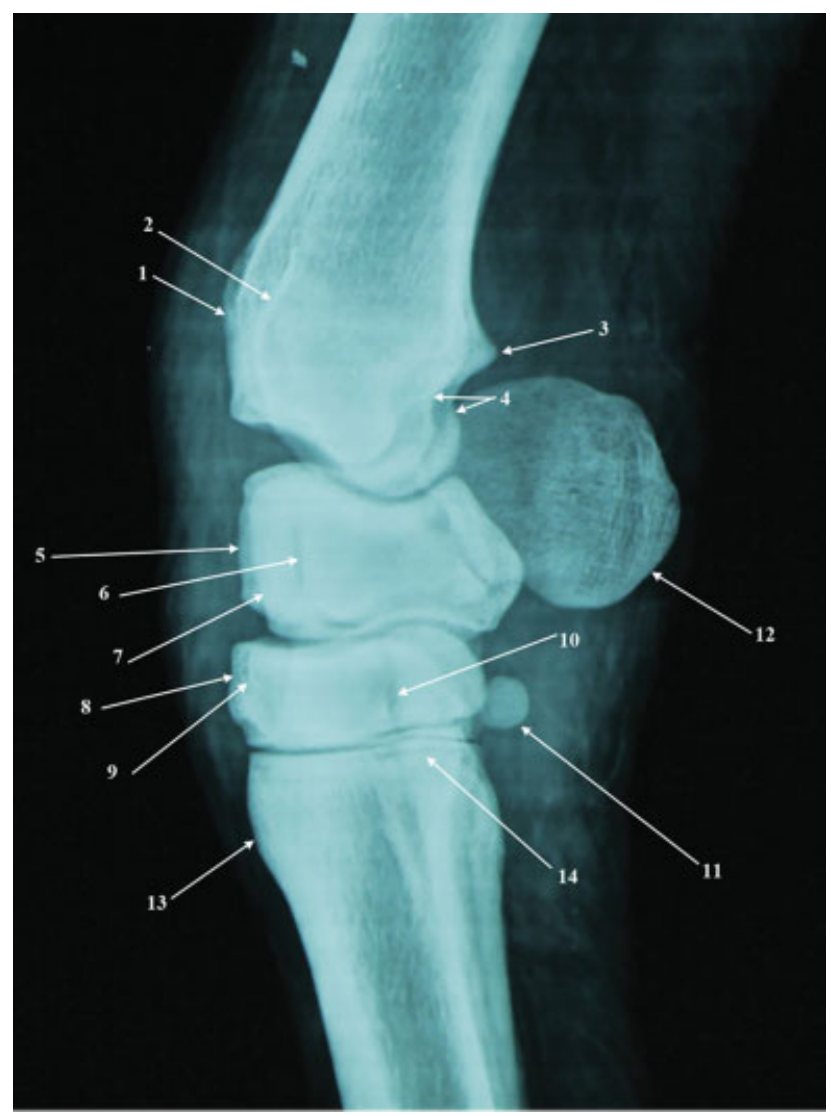

Fig. 2 Lateral radiograph of the left carpal joint. 1. Ridges on the cranial surface of the radius. 2 . Ridge adjacent to the lateral border of the common digital extensor tendon. 3. Transverse crest of the radius. 4. Caudal border of the radial trochlea. 5. Dorsal surface of the intermediate carpal bone. 6 . Dorsal surface of the ulnar carpal bone. 7. Dorsal surface of the radial carpal bone. 8. Dorsal surface of the third carpal bone. 9. Dorsal surface of the fourth carpal bone. 10. Dorsal surface of the second carpal bone. 11. First carpal bone. 12. Accessory carpal bone. 13. Third metacarpal bone. 14 . Second metacarpal bone. 


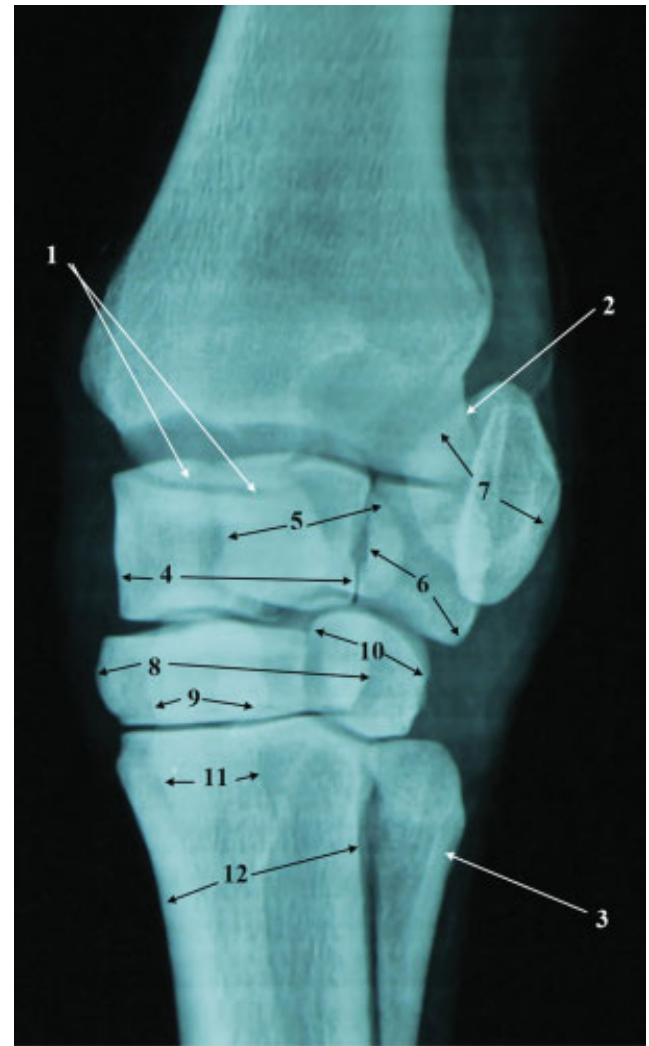

Fig. 3 Dorsolateral-palmaromedial radiograph of the left carpal joint. 1. Medial part of the radial trochlea. 2. Lateral styloid process. 3. Fourth metacarpal bone. 4. Radial carpal bone. 5. Intermediate carpal bone. 6 . Ulnar carpal bone. 7. Accessory carpal bone. 8. Third carpal bone. 9. Second carpal bone. 10. Fourth carpal bone. 11. Second metacarpal bone. 12. Third metacarpal bone.

\section{Computed Tomography}

The radiographically normal and diseased carpal joints were subjected to the bone and soft-tissue window settings. The CT scan was performed in the transverse, sagittal, dorsal and three-dimensional planes. A Toshiba Astesion Super 4 CT scanner with a current of $120 \mathrm{Kv}$ and $112 \mathrm{mAs}$ was used to scan $0.2-\mathrm{mm}$ slices.

\section{Cadaver Cross-sections}

The carpi of the donkey cadaver were frozen under $-20^{\circ} \mathrm{C}$ for one week in extended position; then, $1-\mathrm{cm}$ slices were cut using an electric band saw to correlate them with the CT images of the same joint. The slices were cleaned of debris with water and photographed with the caudal surface facing the camera.

\section{Results}

\section{Radiographic Anatomy of the Carpal Joint}

The dorsopalmar radiographic view of the carpus showed thin radiolucent lines between the articulating surfaces of the carpal bones at the three levels: radiocarpal, middle carpal and carpometacarpal articulations. The radiolucent lines between the individual carpal bones within each row are the intercarpal joints. The fusiform radiolucent zone between the radial and intermediate carpal bones is a normal variant. The intercondylar fossa was detected as a circular radiolucency at the center of the distal extremity of the radius, just above the margin of the joint. The radio-opaque zone that was superimposed laterally on the ulnar carpal bone was the accessory carpal bone. The first carpal bone was visualized as a radio-opaque circular mass behind the second carpal bone. The vascular channels appeared as two tiny radiolucent dots at the middle of distal extremity of the radius, proximal to the carpal articular surface. The medial styloid process appeared medially at the distal extremity of the radius, and it was more prominent than the lateral one (-Fig. 1). The lateral radiographic view of the carpus showed the carpal bones superimposed. The intermediate carpal bone was dorsal, and the dorsal distal border of the radial carpal bone was sharply defined and well fitted within the dorsal proximal surface of the third carpal bone. The distal articular surface of the intermediate carpal bone was more proximal than that of the radial carpal bone. The $3^{\text {rd }}$ carpal bone was prominent, with a little protrusion at the middle of its dorsal surface, and it was partially superimposed caudally with the second and fourth carpal bones(-Fig. 2). The dorsolateral-palmaromedial oblique radiographic view of
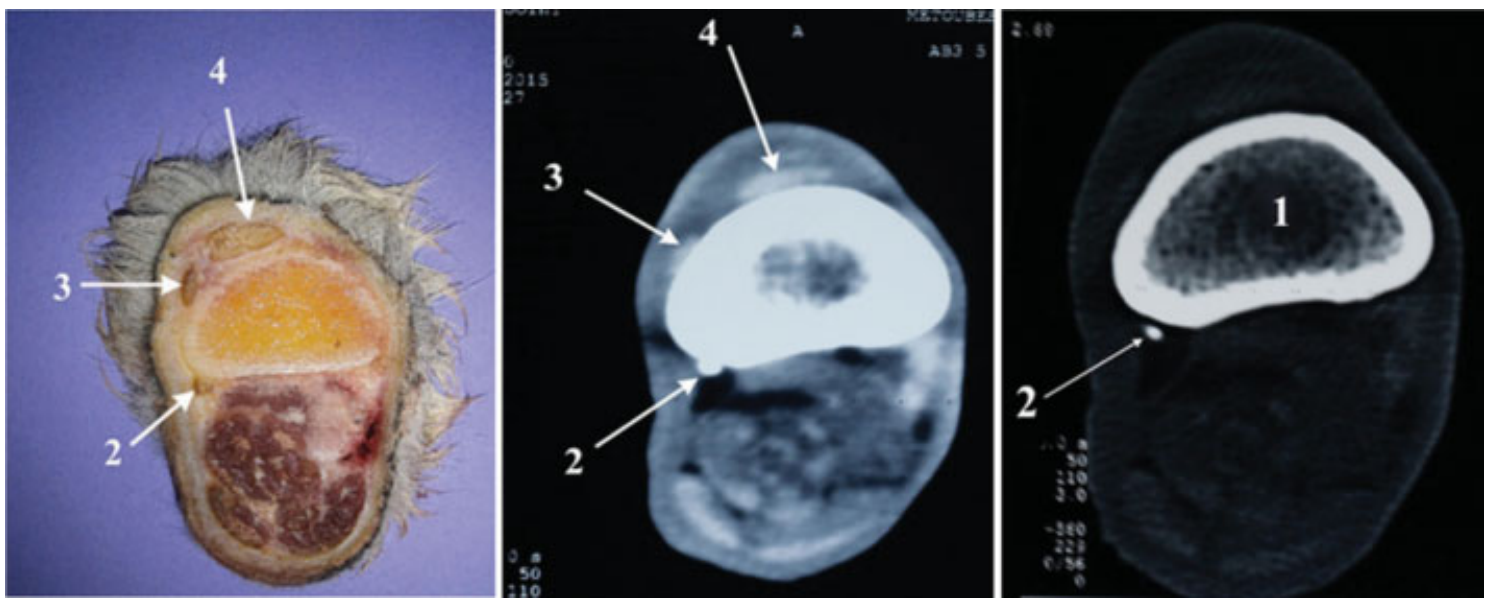

Fig. 4 Cadaver cross-section (A) and computed tomography scans (soft-tissue window [B] and bone window [C]) of the carpal joint at the level of the distal extremity of the radius. 1. Radius. 2. Ulna. 3. Common digital extensor tendon. 4. Extensor carpi radialis tendon. 

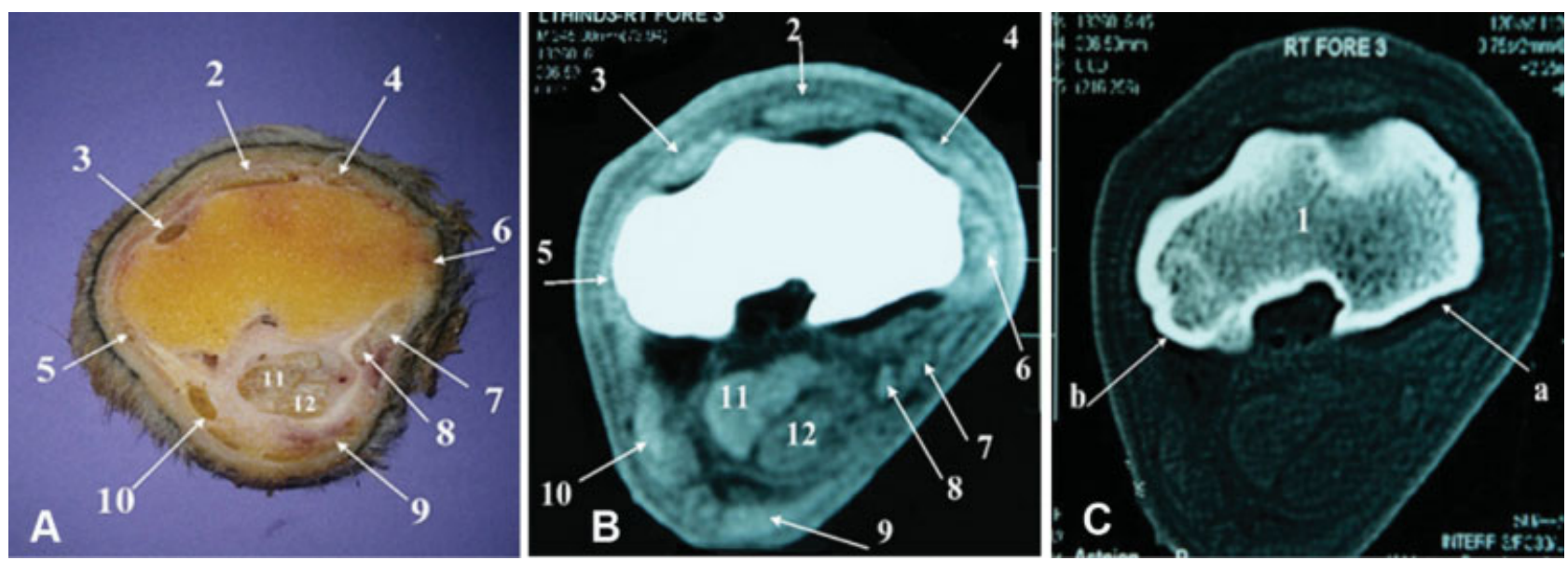

Fig. 5 Cadaver cross-section (A) and computed tomography scans (soft-tissue window $[B]$ and bone window [C]) of the carpal joint at the level of the trochlea radii. 1. Radius (medial aspect [a] and lateral aspect [b]). 2. Extensor carpi radialis tendon. 3. Common digital extensor tendon. 4. Extensor carpi obliquus tendon. 5. Lateral collateral ligament. 6. Medial collateral ligament. 7. Flexor retinaculum. 8. Flexor carpi radialis tendon. 9. Flexor carpi ulnaris muscle (hyperdense tendinous part and hypodense muscle part). 10. Ulnaris lateralis muscle with long tendon (hyperdense). 11. Deep digital flexor tendon. 12. Superficial digital flexor tendon.

the carpus showed the fourth and ulnar carpal bones more clearly than the lateral view. The lateral styloid process was visualized, and it was partially superimposed with the accessory carpal bone. The fourth metacarpal bone was not superimposed, but the second metacarpal bone was totally superimposed with the third metacarpal bone, and articulated with the second carpal bone ( - Fig. 3 ).

\section{Computed Tomograpy Scans of the Normal Carpal Joint}

The bone window showed the bony components of the joint and some soft-tissue components. The soft-tissue window showed the soft tissues much more clearly, and represented the bony components as hyperdense white structures losing their trabecular pattern. The transverse plane at the level of the distal extremity of the radius showed the distal extremity of the radius and the fossa in the middle of its caudal surface, which became clear toward the antebrachiocarpal joint (-Figs. 4-5). The tendon of the extensor carpi radialis appeared on the dorsal surface of the radial trochlea. The superficial and deep digital flexor tendons appeared caudal to the radial trochlea (-Fig. 5). The transverse plane at the level of the proximal carpal row showed the intercarpal joints between the radial, intermediate and ulnar carpal bones. The intercarpal joint between the radial and intermediate carpal bones appeared wider distally (-Fig. 6). The transverse plane at the level of the distal carpal row showed the second, third and fourth carpal bones, while the first carpal bone was behind the second (-Fig. 7).
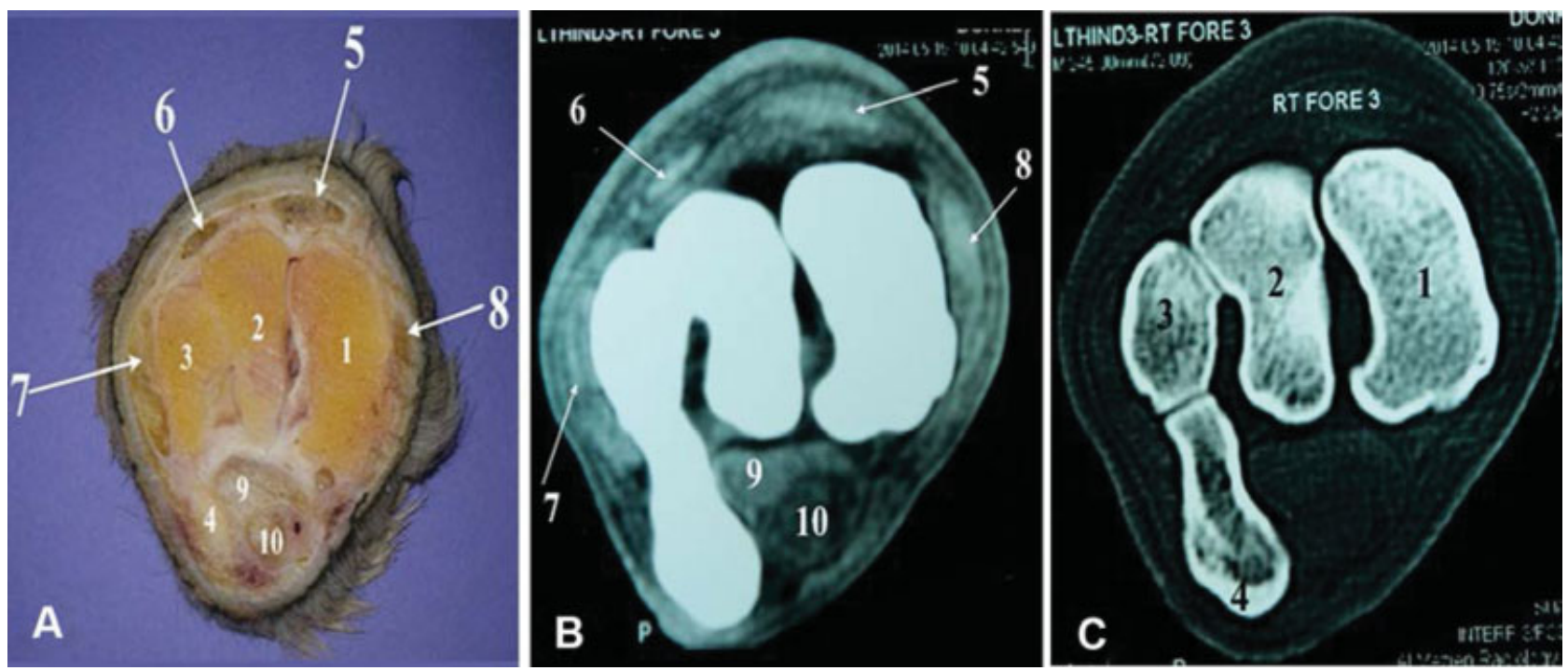

Fig. 6 Cadaver cross-section (A) and computed tomography scans (soft-tissue window [B] and bone window [C]) of the carpal joint at the level of the proximal carpal row. 1. Radial carpal bone. 2. Intermediate carpal bone. 3. Ulnar carpal bone. 4. Accessory carpal bone. 5. Extensor carpi radialis tendon. 6. Common digital extensor tendon. 7. Lateral collateral ligament. 8. Medial collateral ligament. 9. Deep digital flexor tendon. 10. Superficial digital flexor tendon. 

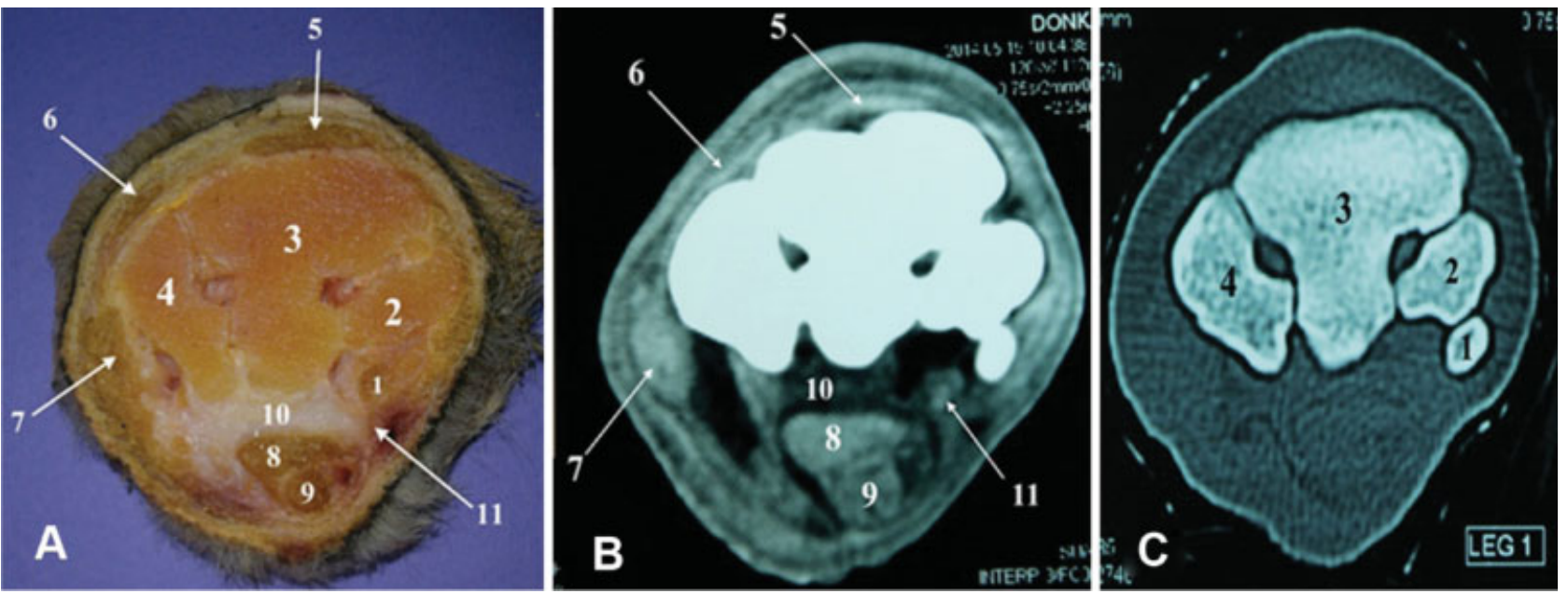

Fig. 7 Cadaver cross-section (A) and computed tomography scans (soft-tissue window [B] and bone window [C]) of the carpal joint at the level of the distal carpal row. 1. First carpal bone. 2. Second carpal bone. 3. Third carpal bone. 4. Fourth carpal bone. 5. Extensor carpi radialis muscle. 6. Common digital extensor tendon. 7. Lateral collateral ligament. 8. Deep digital flexor tendon. 9. Superficial digital flexor tendon. 10. Palmar carpal ligament. 11. Flexor carpi radialis tendon.
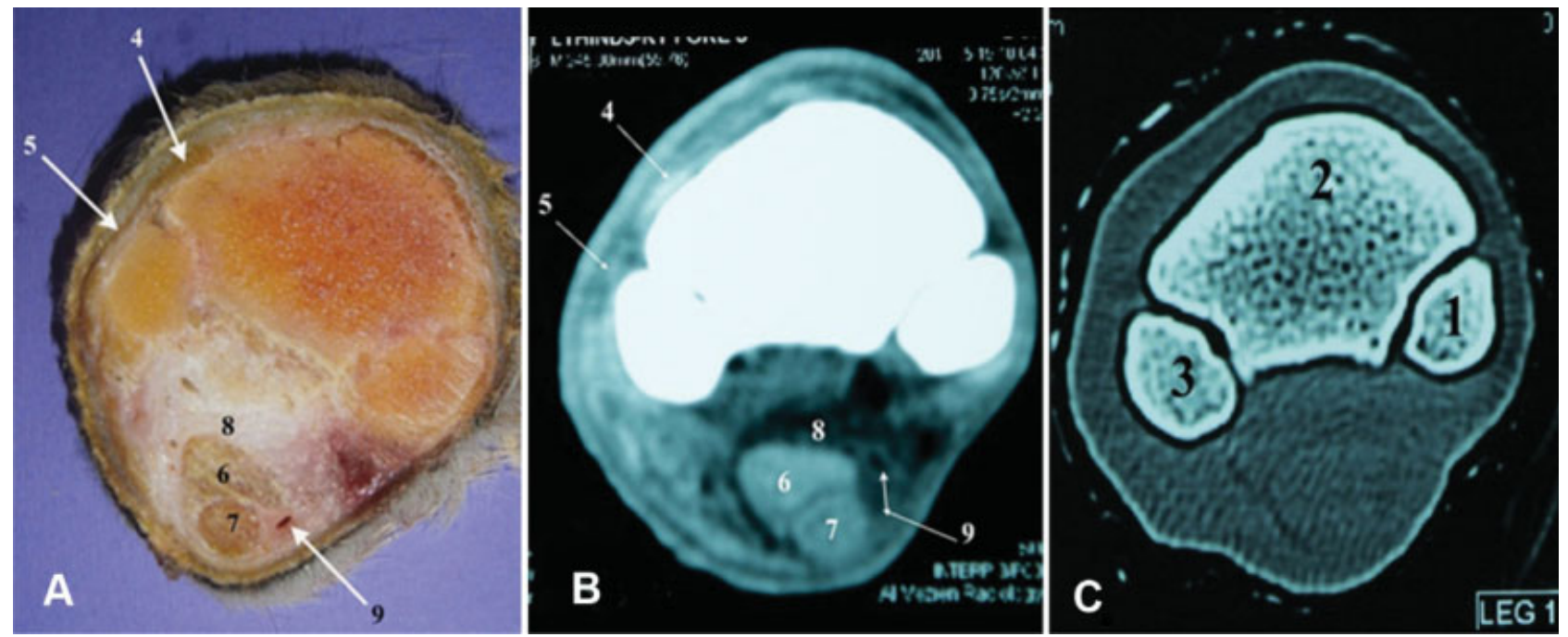

Fig. 8 Cadaver cross-section (A) and computed tomography scans (soft-tissue window [B] and bone window [C]) of the carpal joint at the level of the proximal part of the metacarpus. 1. Second metacarpal bone. 2. Third metacarpal bone. 3. Fourth metacarpal bone. 4. Common digital extensor tendon. 5. Lateral digital extensor tendon. 6. Deep digital flexor tendon. 7. Superficial digital flexor tendon. 8. Accessory ligament of the deep digital flexor tendon. 9. Medial palmar artery.

The transverse plane at the level of the proximal third of the metacarpus showed the second, third and fourth metacarpal bones (-Fig. 8). The sagittal plane showed the lateral,

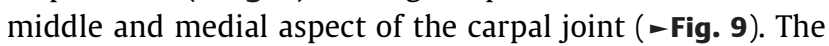
dorsal plane showed the anatomical details of the dorsal, middle and palmar aspects of the carpal joint ( - Fig. 10). The three-dimensional view of the carpal joint showed all the bony prominences and bone alignments (-Fig. 11).

\section{Joint Lesions Detected in CT Scans but Not Detected with X-ray Radiographs}

\section{Bone Cysts}

Bone cysts appeared with a circular or oval shape, regular margins, and surrounded by a sclerotic rim. They appeared in different locations, and had different sizes at the cochlea of the radius bone, second, third, fourth and radial carpal bones (-Figs. 12-13).

\section{Hemorrhagic Bone Cysts (Old Hematomas)}

Hematomas were viewed as irregular radiolucent zones at the distal extremity of the radius bone, and they had different sizes and locations. They resemble the bone cysts, but were characterized by irregular margins and the lack of sclerotic rim. They were detected at the sagittal and dorsal planes (-Fig. 14).

\section{Old Fractures and Microfractures}

Old fractures appeared as small radiolucent lines surrounded by bony sclerosis or thin sclerotic margins. The medial aspect 

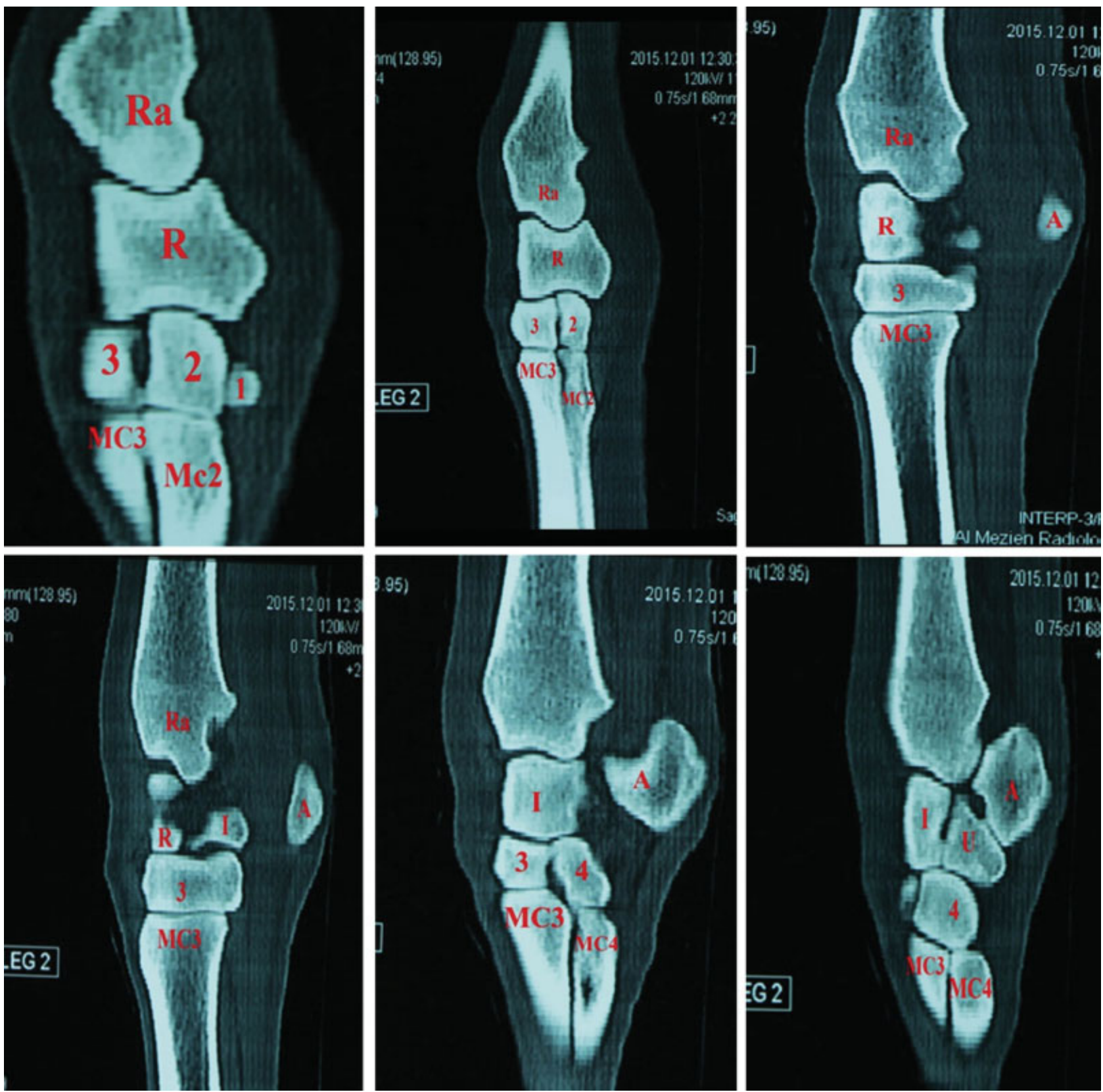

Fig. 9 Sagittal computed tomography scans of the carpal joint from the medial to the lateral aspects. 1. First carpal bone. 2. Second carpal bone. 3. Third carpal bone. Abbreviations: A, accessory carpal bone; I, intermediate carpal bone; MC2, second metacarpal bone; MC3, third metacarpal bone; MC4, fourth metacarpal bone; R, radial carpal bone; Ra, radius; U, ulnar carpal bone.

of the distal extremity of the radius and the radial cochlea were the reported sites of these lesions (-Fig. 15).

\section{Bone Sclerosis}

Bone sclerosis was detected at the radial facet of the third carpal bone. It was characterized by bone sclerosis with increased radiopacity and the loss of the trabecular pattern, with degenerative changes and bone remodeling at its dorsomedial surface ( - Fig. 16).

\section{Normal Anatomical Variations}

The sagittal CT scan of the carpal joint revealed a radiolucent line at the site of the fusion between the radius and the ulna, where the joint was regular, without bone reaction and bone sclerosis around it (-Fig. 17). The transverse and threedimensional CT scans revealed a normal bone protrusion at the middle of the lateral surface of the accessory carpal bone, at the midlevel of the proximal carpal row (-Fig. 18A-B). The lateral radiograph of the carpal joint revealed a normal protrusion at the middle of the dorsal surface of the third carpal bone (-Fig. 18C).

\section{Discussion}

This is the first integrated description of two different radiological techniques applied to normal and pathological 


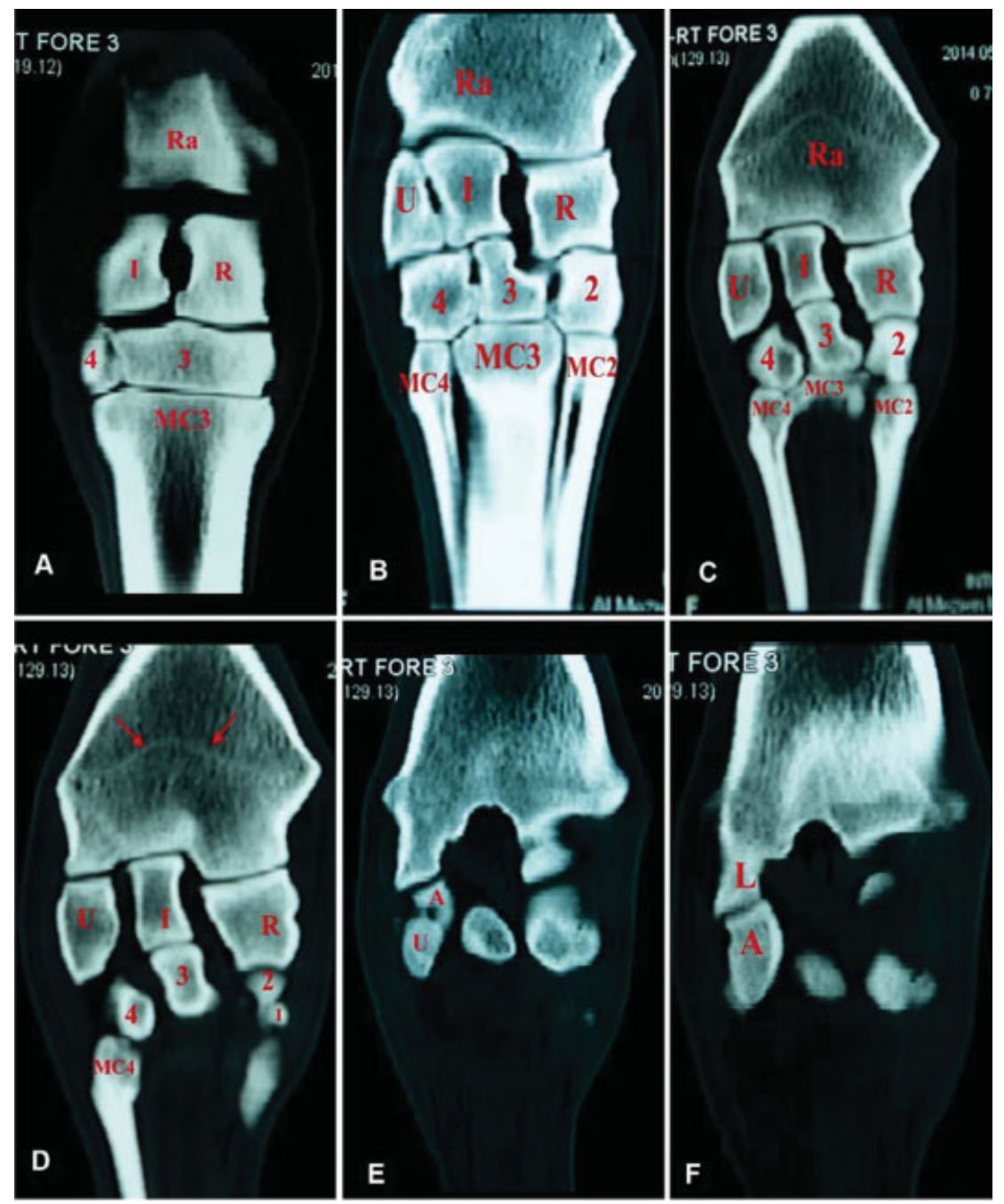

Fig. 10 Dorsal computed tomography scans of the carpal joint from the dorsal to the caudal aspects. (A) Dorsal third. (B) Middle third. (C, D, E, F) Palmar third. 1. First carpal bone. 2. Second carpal bone. 3. Third carpal bone. 4. Fourth carpal bone. Abbrevitions: A, accessory carpal bone; I, intermediate carpal bone; L, lateral styloid process; MC2, second metacarpal bone; MC3, third metacarpal bone; MC4, fourth metacarpal bone; R, radial carpal bone; Ra, radius; U, ulnar carpal bone. The arrows refer to the metaphyseal line.
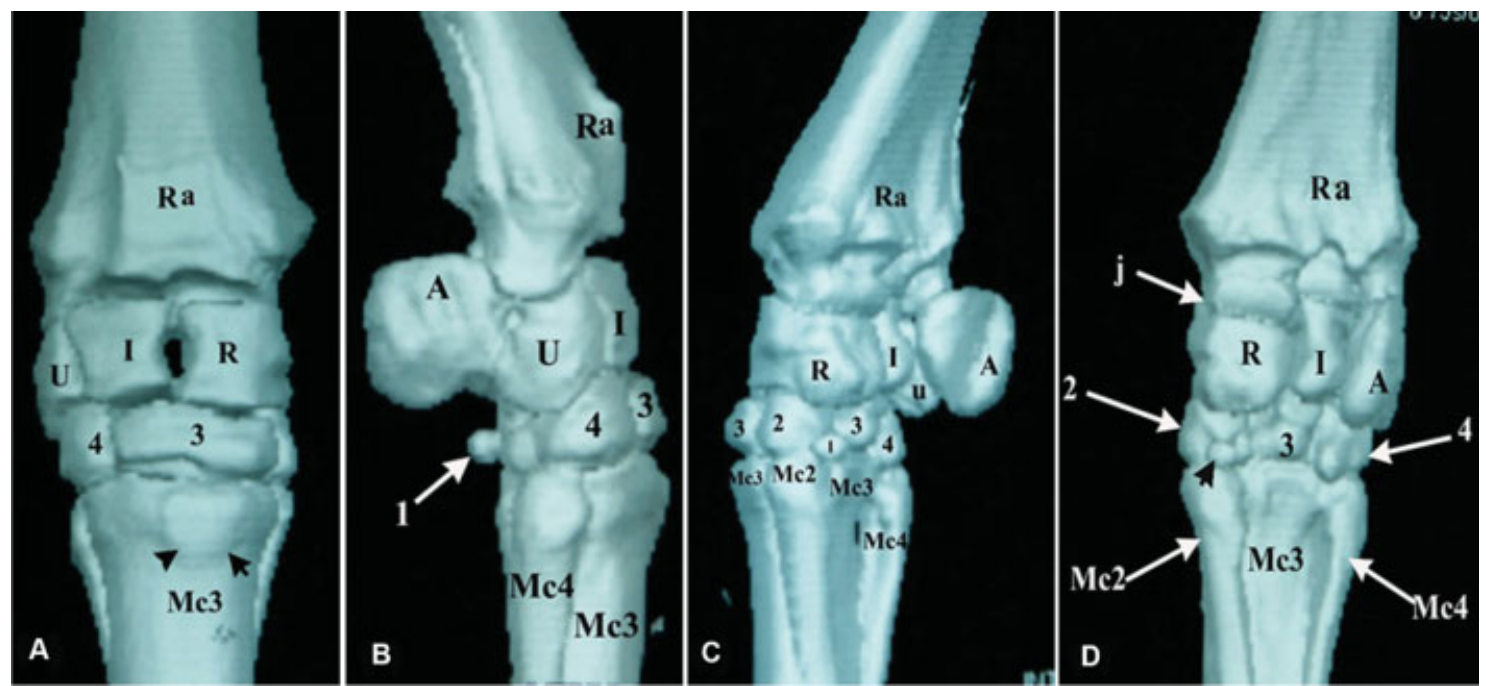

Fig. 11 Three-dimensional computed tomography scan of the carpal joint. (A) Dorsal view. (B) Lateral view. (C) Medial oblique view. (D) Palmar view. 1. First carpal bone. 2. Second carpal bone. 3. Third carpal bone. 4. Fourth carpal bone. Abbreviations: A, accessory carpal bone; I, intermediate carpal bone; MC2, second metacarpal bone. MC3, third metacarpal bone; MC4, fourth metacarpal bone; R, radial carpal bone; Ra, radius; U, ulnar carpal bone. The black arrow refers to the first carpal bone. 

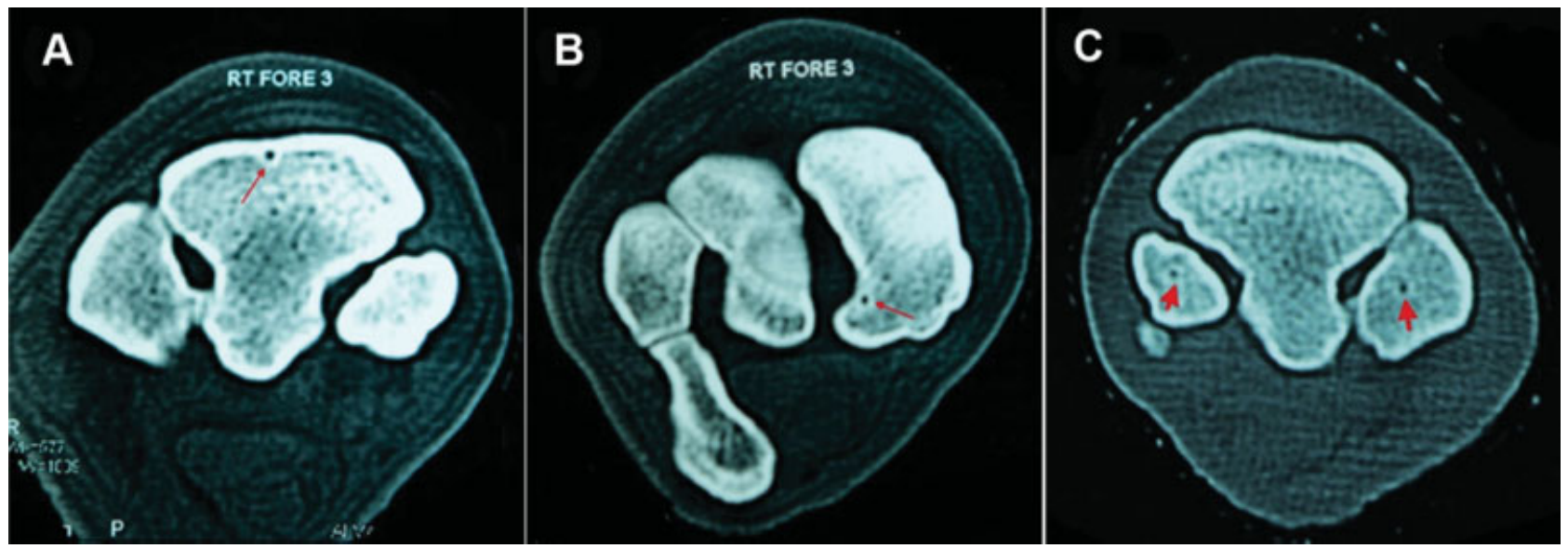

Fig. 12 Transverse computed tomography scans showing bone cysts at the carpal bones. (A) Small bone cyst surrounded by a sclerotic rim at the middle of the dorsal aspect of the third carpal bone. (B) Small bone cyst at the palmaromedial aspect of the radial carpal bone. (C) Small bone cysts at the middle of the second and fourth carpal bones.

carpi of donkeys, and it makes the interpretation of these images by practitioners easier.

Different textbooks ${ }^{8,17,18}$ describe the bones of the carpal joint; in the present study, the bones that constituted the joint and the shape of the first and accessory carpal bones were similar to those of horses. The carpal joint consisted of three articulations: the antebrachiocarpal, middle carpal and carpometacarpal joints. The CT scan of the carpal canal was in line with what was stated in the study by Mcllwraith et al. ${ }^{8}$ The extensor carpi radialis, common digital extensor and extensor carpi obliquus tendons had a bright gray shade on the dorsal surface of trochlea radii, and the accessory ligament of the deep digital flexor tendon appeared hypodense compared with the superficial digital flexor tendon; these findings were similar to those mentioned in the study by Kaser-Hotz et al. ${ }^{13}$

In the present study, the CT scans revealed that bone cysts had a circular or oval shape, regular margins, and were surrounded by sclerotic rims, which were observed in different locations and had different sizes; these findings matched those described by Bergman and Saunders. ${ }^{15}$ The findings reported in the present study about the places of the subchondral or osseous cyst-like lesions observed at the carpal bones and at the distal aspect of the radius were similar to the findings reported by Thrall. $^{19}$ The small bone cysts detected at the carpal joint and carpal bones were not visible in any of the radiographic views in the current study; this
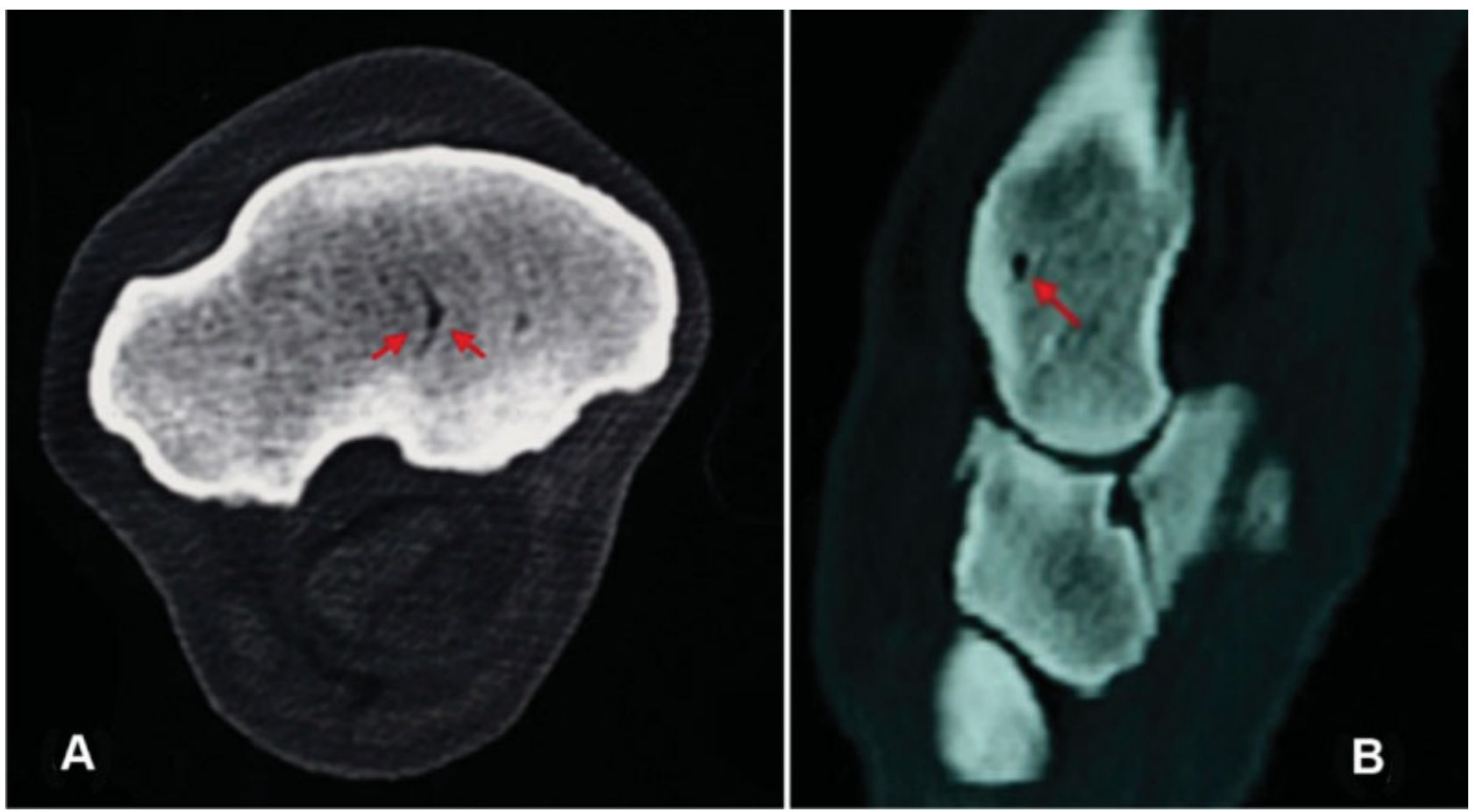

Fig. 13 Computed tomography scans showing bone cysts at the distal extremity of the radius. (A) Elongated bone cyst at the middle of the trochlea radii. (B) Bone cyst at the lateral side of the distal extremity of the radius, close to the carpal joint. 


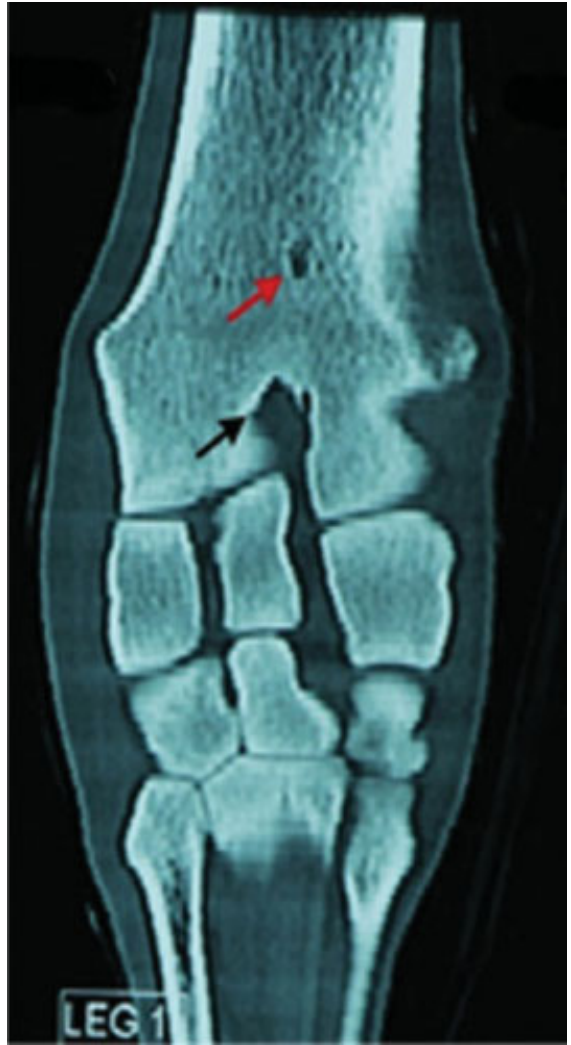

Fig. 14 Dorsal computed tomography scans of the carpus showing irregular hematoma at the middle of the palmar aspect of the distal extremity of the radius (red arrow). The black arrows refer to the normal intercondylar fossa. may be due to a fact mentioned by Greenfield, ${ }^{20}$ that 30 to $40 \%$ of change in bone mineral density is required before bone changes can be appreciated radiographically. The CT without contrast gave precise information about the location and extent of the lesion, but it did not enable the assessment of the integrity of the cartilage. ${ }^{21}$ The fractures reported in the carpal joint in the medial aspect of the distal extremity of the radius, especially at the radial cochlea, after the CT scan had the same description mentioned by others authors; ${ }^{22,23}$ these fractures were not detected in any of the radiographic views used in the present study. The site of the bone sclerosis at the carpal joint detected at the radial facet of the third carpal bone was characterized by high radiopacity and loss of the trabecular pattern, with degenerative changes and bone changes at its dorsomedial surface; these findings are in line with findings previously recorded in the study by Bergman and Saunders. ${ }^{15}$

\section{Conclusion}

In the present study, we provide a detailed radiographic and computed tomographic anatomical reference for the donkey carpus. Both imaging techniques are suitable for the examination of the carpus, and the selection of the technique is conditioned to many factors, like the type of tissue affected, and maybe economic reasons, such as the availability of the apparatus and the cost of the animal. Expensive techniques are more suitable for expensive animals like racehorses, but they are difficult to use in normal working donkeys.
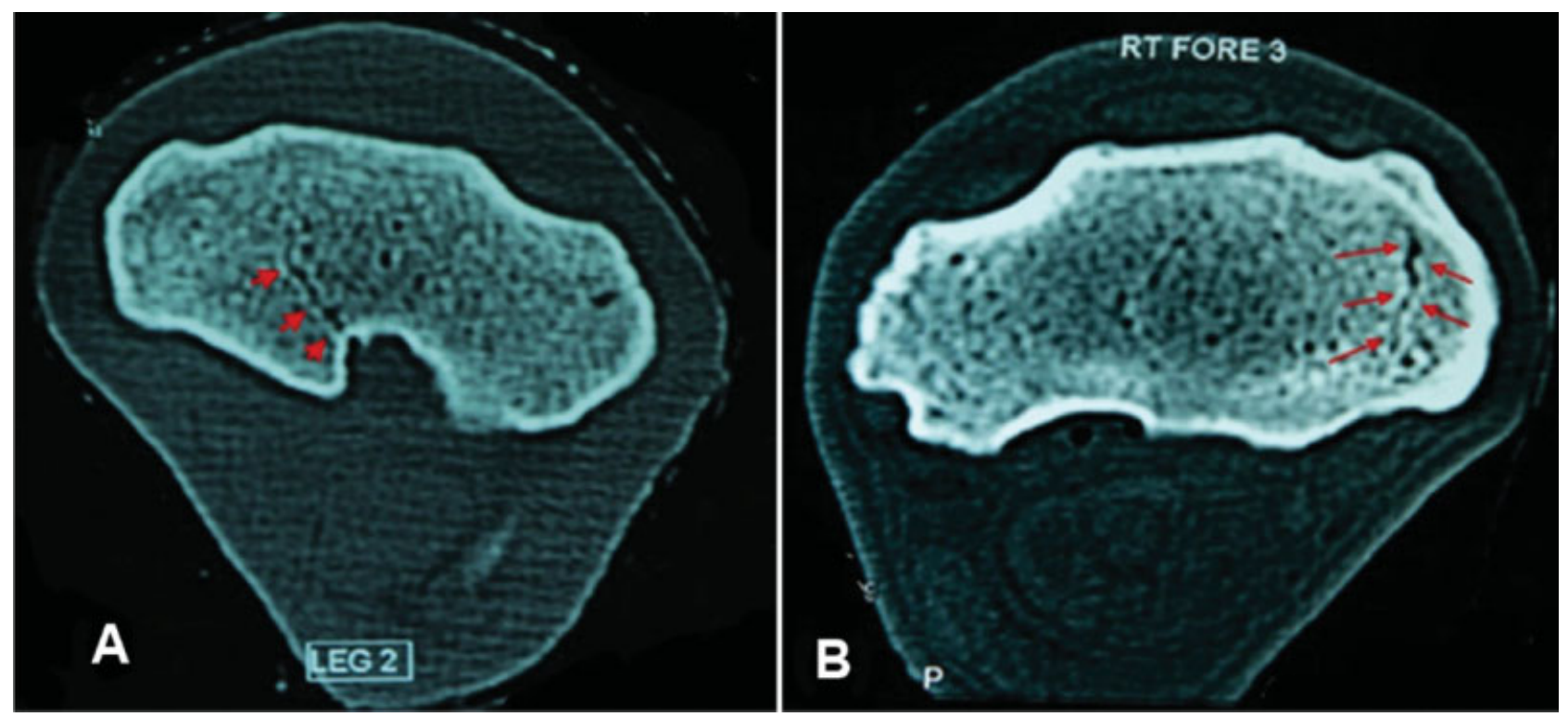

Fig. 15 Transverse computed tomography scan showing an old fracture. (A) Thin radiolucent line surrounded by a sclerotic rim at the level of the trochlea radii of the palmaromedial aspect of the left carpal joint. (B) Old fracture at the medial aspect of the distal radius at the level of the distal extremity of the radius in the right carpal joint. 


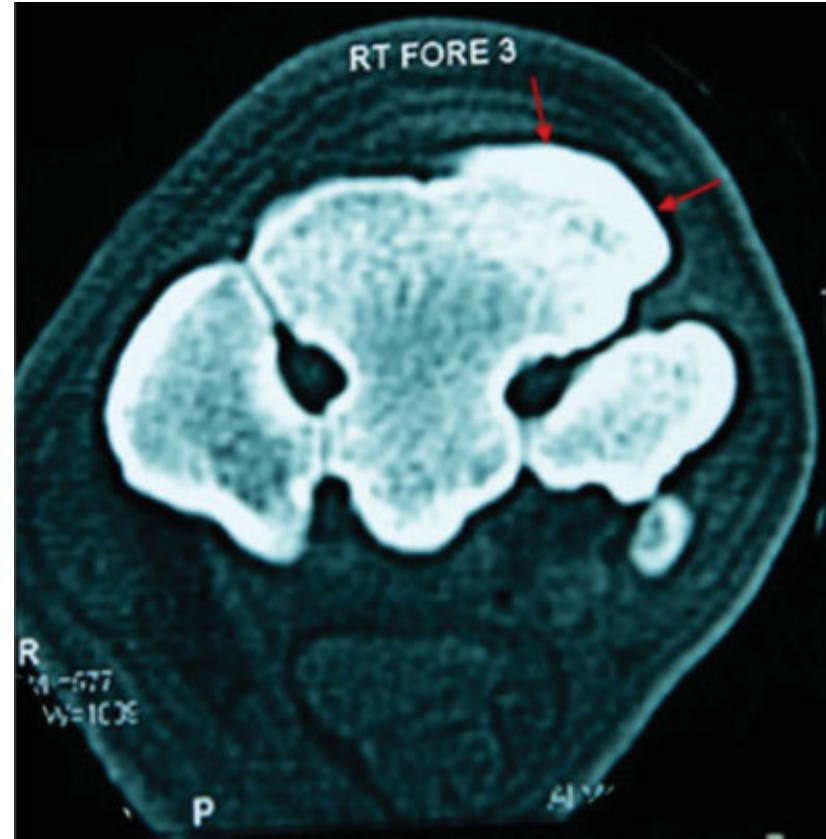

Fig. 16 Transverse computed tomography scan of the third carpal bone showing bony sclerosis at the radial facet with degenerative changes and bone remodeling at the dorsomedial surface.

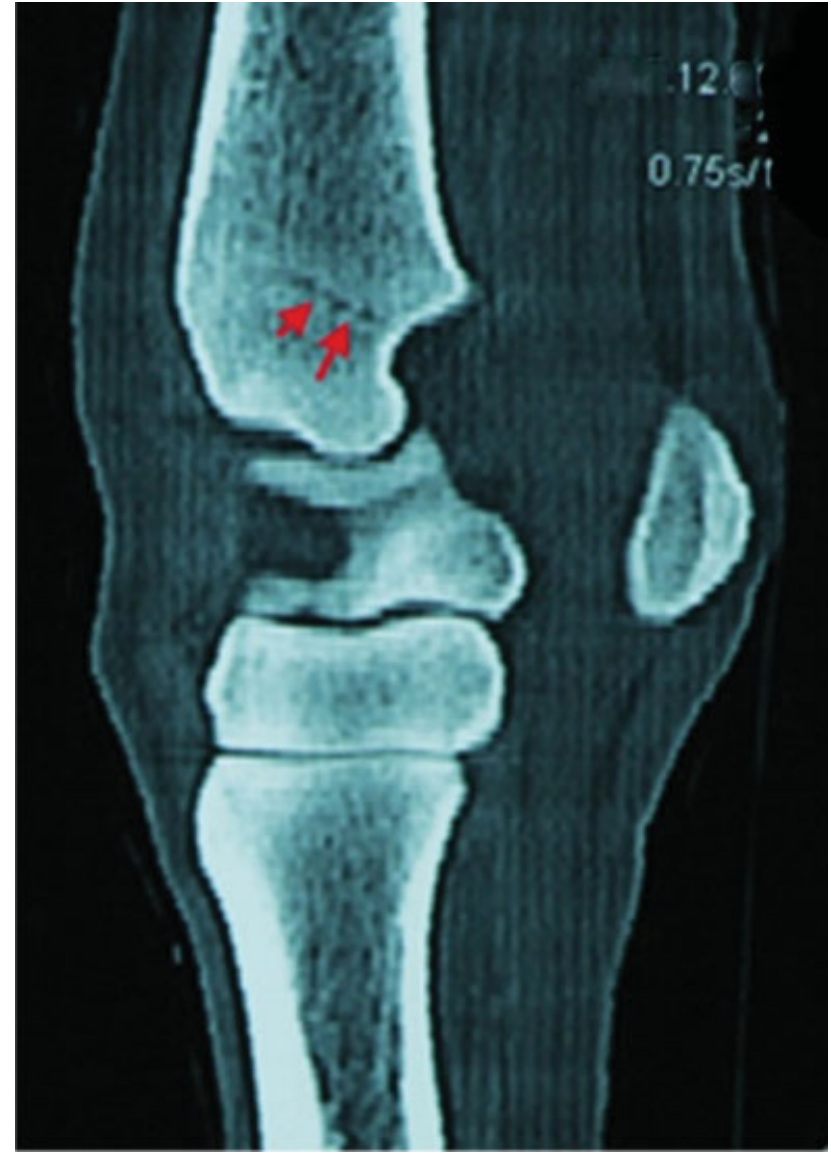

Fig. 17 Sagittal computed tomography scans of the lateral third of the carpal joint showing incomplete ossification at the line of the fusion of the radius and ulna.
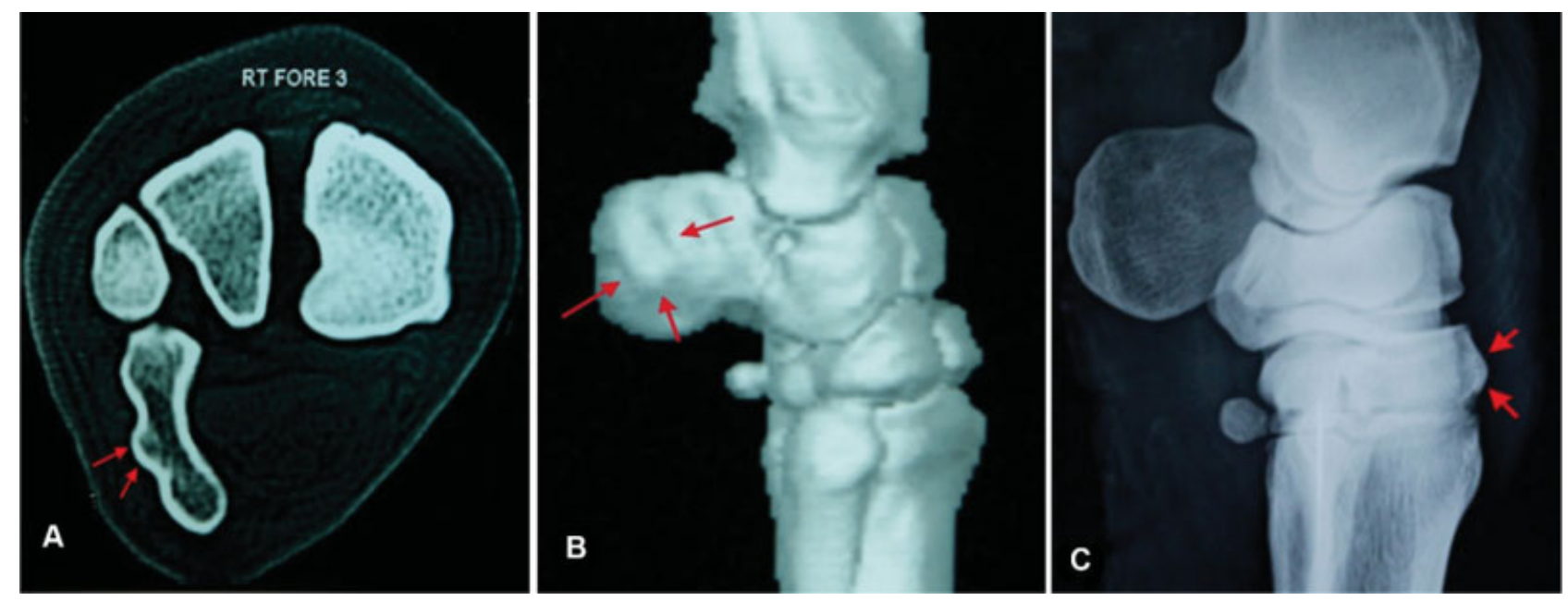

Fig. 18 A normal anatomic variant at the carpal joint. (A) Transverse computed tomography (CT) scan. (B) Three-dimensional CT showing a protrusion at the middle of the lateral surface of the accessory carpal bone. (C) Lateral radiographic view showing a normal protrusion at the dorsal surface of the third carpal bone. 


\section{Conflict of Interests}

The authors have none to disclose.

\section{References}

1 Marshall K, Ali Z. Gender issues in donkey use in rural Ethiopia. Donkeys, People and Development, a Resource Book of the Animal Traction Network for Eastern and Southern Africa (ATNESA), 1997:62-68

2 El-Gendy SA, Alsafy MA, El Sharaby AA. Computed tomography and sectional anatomy of the head cavities in donkey (Equus asinus). Anat Sci Int 2014;89(03):140-150

3 Dik K, Weller R, Saunders J, et al. Diagnostic Imaging of Equine Sport Injuries. In Nuclear Medicine and Radiologic Imaging in Sports Injuries. 2015Springer

4 Pool R. Pathologic manifestations of joint disease in the athletic horse. In Joint disease in the horse 1996;6:87-103

5 Todhunter R, Lust G. Pathophysiology of synovitis: clinical signs and examination in horses. The Compendium on continuing education for the practicing veterinarian, 1990; (USA)

6 Baxter G, Stashak T. Examination for lameness, 2011; WileyBlackwell, UK, USA.

7 Park RD, Steyn PF, Wrigley RH. Imaging techniques in the diagnosis of equine joint disease. In: Joint Disease in the Horse, Eds: C.W. Mcllwraith and C.W. Trotter, 1996W.B. SaundersPhiladelphia

8 Mcllwraith CW, Frisbie DD, Kawcak CE, van Weeren R. Joint disease in the horse. 2015Elsevier Health Sciences

9 Vanderperren K, Saunders JH. Diagnostic imaging of the equine fetlock region using radiography and ultrasonography. Part 1: Soft tissues. Vet J 2009;181(02):111-122

10 Semieka MA, Ali MM. Radiography of Manus and Pes in hard working donkeys. J Adv Vet Res 2012;2:32-37
11 Burguez PN. Interpreting radiographs 4: The carpus. Equine Vet J 1984;16(03):159-162

12 Tnibar M, Kaser-Hotz B, Auer JA. Ultrasonography of the dorsal and lateral aspects of the equine carpus: technique and normal appearance. Vet Radiol Ultrasound 1993;34:413-425

13 Kaser-Hotz B, Sartoretti-Schefer S, Weiss R. Computed tomography and magnetic resonance imaging of the normal equine carpus. Vet Radiol Ultrasound 1994;35:457-461

14 Badawy AM. Computed Tomographic anatomy of the fore foot in one-humped camel (Camelus dromedarius). Glob Vet 2011; 6:417-423

15 Bergman HJ, Saunders J. Equine upper limbs (carpus, tarsus, stifle). Vet Comp Tom 2011:483-501

16 Raji AR, Sardari K, Mohammadi HR. Normal cross-sectional anatomy of the bovine digit: comparison of computed tomography and limb anatomy. Anat Histol Embryol 2008;37(03):188-191

17 Dyce KM, Sack WO, Wensing CJG. Textbook of Veterinary Anatomy. 2010Mosby

18 König HE, Liebich H-G. Anatomie der Haustiere. 2019Georg Thieme VerlagStuttgart

19 Thrall DE. Textbook of veterinary diagnostic radiology. 2013Elsevier Health Sciences

20 Greenfield GB. Radiology of bone disease. 1986Lippincott Williams and WilkinsUSA

21 Zanon G, DI Vico G, Marullo M. Osteochondritis dissecans of the talus. Joints 2014;2(03):115-123

22 Murphey ED, Schneider RK, Adams SB, Santschi EM, Stick JA, Ruggles AJ. Long-term outcome of horses with a slab fracture of the central or third tarsal bone treated conservatively: 25 cases (1976-1993). J Am Vet Med Assoc 2000;216(12):1949-1954

23 Vedrine B. Comminuted fracture of the ulnar carpal bone in a Labrador retriever dog. Can Vet J 2013;54(11):1067-1070 